\title{
Spatiotemporal factors and developmental changes in visual processes
}

\author{
FRED L. ROYER \\ Cleveland Veterans Administration Medical Center, Brecksville, Ohio \\ and \\ GROVER C. GILMORE \\ Case Western Reserve University, Cleveland, Ohio
}

\begin{abstract}
Contrast sensitivity functions were measured in elderly and young persons for sinusoidal gratings that reversed phase by $180^{\circ}$ at rates of 0.3 and $6.3 \mathrm{~Hz}$. Elderly subjects showed less sensitivity than young subjects for spatial frequencies. Their sensitivity to high spatial frequencies declined faster as the rate of reversal increased. These changes in sensitivity with age indicate a differential increase in the integration time constants of transient and sustained channels.
\end{abstract}

Spatial sensitivity of the visual system changes with age; sensitivity to high spatial frequencies in static displays decreases with age (Kline, Schieber, Abusamra, \& Coyne, 1983; Owsley, Sekuler, \& Siemsen, 1983). As Kline and Schieber (1981) suggested, these changes may reflect aging of neural systems that are differentially sensitive to spatial extent (spatial frequency) and rapidity of change in stimulation (temporal frequency). Both psychophysical evidence and neurophysiological evidence point toward the existence of two visual neural subsystems, called transient and sustained channels (see reviews by DeValois \& DeValois, 1980, and by Sekuler, 1974). The channels differ in sensitivity to spatial and temporal frequencies. Transient channels are sensitive to low spatial frequencies and high temporal frequencies. Sustained channels are sensitive to high spatial frequencies and low temporal frequencies.

On the basis of work by Sekuler and his colleagues (Sekuler \& Hutman, 1980; Sekuler, Hutman, \& Owsley, 1980), showing that sensitivity to low spatial frequencies was reduced in elderly subjects, Kline and Schieber (1981) hypothesized that sustained and transient channels age at different rates.

Kline et al. (1983) found that the greatest loss of contrast sensitivity for stationary gratings in aging was in intermediate and high spatial frequencies, from 4 cycles per degree (cpd) and up, suggesting an age-related decline in the sustained channels, not in the transient ones. Owsley et al. (1983) also showed a loss of sensitivity at high frequencies with stationary gratings; however, they found

\footnotetext{
This research was supported by NIA Grant R01 AG03178 and by the Veterans Administration Medical Research Service. The authors acknowledge the special contribution of Charlotte Andrist in overseeing the many daily details of enlisting, scheduling, and testing subjects.

Requests for reprints should be addressed to G. C. Gilmore, Department of Psychology, Case Western Reserve University, Cleveland, $\mathrm{OH}$ 44106.
}

a substantial age-related decline in sensitivity for a 1-cpd grating as movement increased from 1.1 to $4.3 \% / \mathrm{sec}$. It is the transients which are most sensitive to moving and flickered stimuli. Therefore, the data suggest a decline in function of transients also.

One needs to obtain spatiotemporal sensitivity functions to better understand these problems. We measured contrast sensitivity for slowly and rapidly flickering sinusoidal gratings in young and elderly subjects in which the flickering was accomplished by $180^{\circ}$ phase reversal. That is, the light bars of the grating abruptly became dark and the dark bars abruptly became light. As Mackay (1969) pointed out in the context of evoked cortical potentials to pattern reversals, the $180^{\circ}$ phase reversal is a limiting case of infinitely rapid motion; thus, this method is more likely than either motion or counterphase modulation to maximize activation of transient channels. Contrast sensitivity should be greater for low than high spatial frequencies at the higher flicker rate, and sensitivity for high frequencies should decrease as the flicker rate increases (Regan, 1981). Optimal evoked potentials in the low frequency region are found around a $6-\mathrm{Hz}$ reversal rate.

\section{METHOD}

\section{Subjects}

There were 15 young and 15 elderly subjects in this study. The mean age of the young group was 21.1 years and that of the elderly group was 69.4 years. The mean scaled scores of the Wechsler Adult Intelligence Scale (WAIS) Vocabulary subtest were 14.5 for the young and 14.1 for the elderly group. The mean educational level of the young group was 15.5 years and of the elderly group was 15.2 years. It is clear that the groups had comparable educational and intellectual levels. All elderly subjects were in good health by their report, lived independently in the community, and were socially active. The young subjects were students or staff at the University; the elderly subjects were drawn largely from volunteer workers at University Hospitals and their friends. All subjects had uncorrected or corrected visual acuity of $20 / 30$ or better. Potential subjects having any history of eye disease were rejected from the study. 


\begin{abstract}
Apparatus
Sinusoidal gratings were displayed on a Hewlett-Packard 1332A X-Y Display Monitor. The gratings were generated by means of a microprocessor controlled system built by Fritsch and Keck. Mean luminance was $21.24 \mathrm{~cd} / \mathrm{m}^{2}$. Maximum contrast was $56.6 \%$. The subject controlled the contrast of the display by pressing buttons that increased or decreased contrast. The display was $4^{\circ}$ of visual angle wide and $3.2^{\circ}$ high at a viewing distance of $170.5 \mathrm{~cm}$. The room was dark except a small masked low intensity light at the grating generator console, screened from the subject's view, provided light to record the responses. Both subject and experimenter were dark adapted for at least $5 \mathrm{~min}$ before measurements were made.
\end{abstract}

\section{Procedure}

Thresholds were obtained by the method of limits. The display was previewed at maximum contrast. Then contrast of the display was set to zero (i.e., mean luminance). The subject pushed one button to increase the contrast of the display until the flickering bars were barely visible. Then the subject pushed another button to decrease the contrast until the bars were no longer visible. Practice trials were given before measurements were made. The threshold was taken as the mean of five ascending and five descending threshold measurements. Viewing was through normal pupils.

\section{Design}

Displays of 5 different spatial frequencies were presented: $.5,1,4$, 8 and $12 \mathrm{cpd}$. The gratings were presented at two different frequencies of reversals: 0.3 and $6.3 \mathrm{~Hz}(0.6$ and 12.6 reversals per sec). Thus, the design was groups $(2) \times \mathrm{CPD}(5) \times$ temporal frequency $(2)$.

\section{RESULTS}

In the analysis of variance of sensitivities, significant effects were found for spatial frequency $[\mathrm{F}(4,112)=$ $211.59, \mathrm{p}<.0001]$, and the interaction of spatial frequency $\times$ temporal frequency $[\mathrm{F}(4,112)=23.36, \mathrm{p}<$ $.0001]$. These effects are expected on the basis of prior work (Regan, 1981).

There was a significant effect for age group $[F(1,28)$ $=61.75, \mathrm{p}<.0001]$. The mean sensitivity of the older group, 41.4, was less than that of the younger, 77.9. These means are of little practical consequence for understanding the pattern of change in sensitivity due to aging, but they do indicate that sensitivity is reduced significantly in older subjects over all frequencies. However, significant interactions were found for the effects of spatial frequency $\times$ age groups $[\mathrm{F}(4,112)=8.77, \mathrm{p}<$ $.0001]$, and of temporal frequency $\times$ spatial frequency $\times$ age groups $[F(4,112)=2.91, p=.02]$. The interaction is shown graphically in Figure 1. These interactions are not easily attributable to changes in the optics of the eye. Furthermore, Owsley et al. (1983) measured sensitivity of moving gratings in young subjects with a neutral density filter that reduced retinal illuminance to a level comparable to that expected in older subjects. The filter had no effect; they concluded that "the low level of temporal enhancement in observers in their 60 s is not attributable to reduced retinal illuminance" (Owsley et al., 1983 , p. 696) that would be attributed to optical changes.

\section{DISCUSSION}

While there are differences between age groups at every spatial frequency, the greatest differences in sensitivity to phase reversal gratings

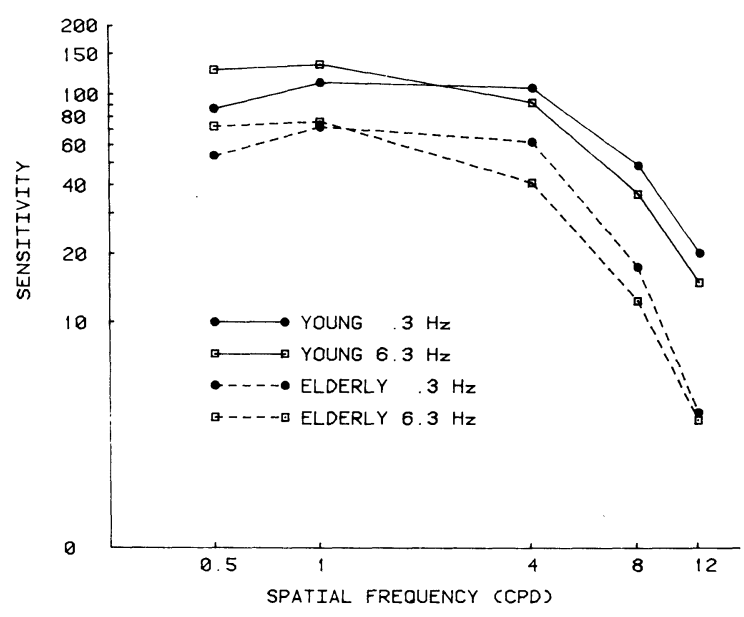

Figure 1. Contrast sensitivity of elderly and young groups as a function of spatial frequency and rate of $180^{\circ}$ phase reversal of vertical sinusoidal gratings.

between the two groups are in intermediate and high spatial frequencies. The attenuation of sensitivity for high spatial frequencies, which occurs as temporal frequency increases, is more rapid in elderly than young subjects. This finding is inconsistent with that of Sekuler and Hutman (1980) who found reduced sensitivity only in the low spatial frequencies. Our results are consistent with the findings of others for static gratings, however.

The curves for the elderly subjects show a faster drop in sensitivity at the upper spatial frequencies and particularly in the fast temporal condition. One hypothesis is that there is an age-related increase in the time constant of visual integration. An increase in the integration time constant of the sustained channels with aging should produce the observed rapid roll-off of sensitivity functions as temporal frequency increases. The gratings were phase reversed periodically by $180^{\circ}$. This reversal is analogous to the square-wave (on-off) modulation of a spot of light in a critical flicker-fusion frequency (CFF) paradigm. It is well known that CFF decreases with age (see review by Corso, 1981). At certain frequencies, the nervous system smoothes the variation, integrating the stimulus over some time period. A fused single stimulus is seen. The same smoothing of luminance changes occurs in phase-reversed gratings at low contrast. A suprathreshold stationary grating may disappear when phase reversal occurs. When gratings are flickered, one observes that the integration time constant varies with spatial frequency. The increased time constant in the elderly would be expected in both transient and sustained channels, given the findings here. The constant would be different however, for the two channels. Kline and Schieber (1981) suggested that transient and sustained channels age at different rates. Alternatively, both age at the same rate, but the time constants of the spatiotemporal tuning functions of the two types of channels both undergo an increase with aging, but the increase is proportional to the channels' sensitivity before aging.

Whether or not the observations are wholly or partly due to changes in optical properties of the eye or changes in neural functioning, they demonstrate the existence of individual differences capable of clarifying age-related changes in performance on perceptual tasks that demand the processing of dynamic visual information at mesoptic illumination levels.

\section{REFERENCES}

CoRso, J. F. (1981). Aging, sensory systems, and perception. New York: Praeger.

DeValois, R. L., \& DeValois, K. K. (1980). Spatial vision. Annual Review of Psychology, 31, 309-342. 
KLINE, D. W., \& SChieber, F. (1981). Visual aging: A transient/sustained shift? Perception \& Psychophysics, 29, 181-182.

Kline, D. W., Schieber, F., Abusamra, L. C., \& Coyne, A. C. (1983). Age, the eye, and the visual channels: contrast sensitivity and response speed. Journal of Gerontology, 38, 211-216.

MACKAY, D. M. (1969). Evoked brain potentials as indicators of sensory information processing. Neurosciences Research Program Bulletin, 7, 3.

Owsley, C., Sekuler, R., \& Siemsen, D. (1983). Contrast sensitivity throughout adulthood. Vision Research, 23, 689-699.

REgAN, D. (1981). Evoked potential studies of visual perception. Canadian Journal of Psychology, 35, 77-112.
SEKULER, R. (1974). Spatial vision. Annual Review of Psychology, 25 , 195-232.

Sekuler, R., ' \& Hutman, L. P. (1980). Spatial vision and aging: I. Contrast sensitivity. Journal of Gerontology, 35, 700-706.

Sekuler, R., Hutman, L. P., \& OwSley, C. J. (1980). Human aging and spatial vision. Science, 209, 1255-1256.

(Manuscript received for publication April 8, 1985.) 